\title{
The Relationship of Ki-67 Over-expression with Clinicopathological Prognostic Parameters in Invasive Breast Carcinomas
}

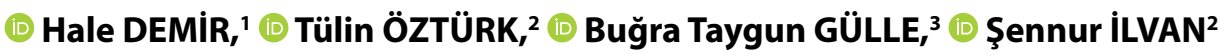 \\ 'Department of Pathology, Amasya University Faculty of Medicine, Amasya-Turkey \\ ${ }^{2}$ Department of Pathology, Istanbul University-Cerrahpasa Faculty of Medicine, Istanbul-Turkey \\ ${ }^{3}$ Department of Public Health, Merzifon District Health Directorate, Amasya-Turkey
}

\begin{abstract}
OBJECTIVE
Immunohistochemical Ki-67 index is a useful method to determine the prognosis. We aimed to evaluate the association of Ki-67 score, using $14 \%$ and $20 \%$ cut-off values, with clinicopathological parameters in invasive breast carcinomas.
\end{abstract}

\section{METHODS}

Pathology reports of 162 females were retrospectively reviewed and parameters including age, menopausal status, multifocality/multicentricity (MF/MC), tumor size, histological type, grade, lymphovascular invasion (LVI), perineural invasion, axillary lymph node status, ER, PR, HER2 status, Ki-67 index, and molecular subtype were recorded. The cases were grouped according to two separate Ki-67 cut-off values (high: $\geq 14 \%$ and $\geq 20 \%$, low $<14 \%$, and $<20 \%$ ). Ki-67 score was compared with other clinicopathological parameters statistically using Chi-square test.

\section{RESULTS}

When the Ki-67 score was grouped according to $14 \%$ or $20 \%$ cut-off values, it was found to be associated with similar clinicopathological parameters. There was a significant correlation between high Ki-67 score and high grade $(\mathrm{p}<0.001, \mathrm{p}<0.001)$, LVI $(\mathrm{p}=0.002, \mathrm{p}=0.022)$, ER negativity $(\mathrm{p}=0.001, \mathrm{p}<0.001)$. When ER expression was grouped as negative, low positive and positive, similar results were obtained $(\mathrm{p}=0.003, \mathrm{p}=0.001)$. There was a significant association between Ki-67 score and molecular subtypes $(\mathrm{p}<0.001, \mathrm{p}<0.001)$ : Ki-67 score was higher in cases that belong to Luminal B subtype and lower in cases that belong to Luminal A in comparison to others. Ki-67 score had no association with age, menopausal status, MF/MC, tumor size, perineural invasion, axillary lymph node involvement, PR, and HER2 status.

\section{CONCLUSION}

Standardization of interpretation of Ki-67 proliferative index and cut-off value for scoring will improve the demonstration of the prognostic signification of Ki-67 in invasive breast carcinomas.

Keywords: Breast cancer; Ki-67; molecular subtype; prognosis.

Copyright $\odot$ 2021, Turkish Society for Radiation Oncology

\section{Introduction}

$\mathrm{Ki}-67$ is a proliferation marker that controls the cell cycle. It is expressed in the cell nucleus and reaches the peak level during mitosis.[1] Tumors that exhibit increased proliferation tend to be more aggressive clinically and immunohistochemical detection of the Ki-67 index is a useful method to determine the prognosis. 
[1-3] It was reported that the high Ki-67 levels were associated with decreased survival in patients with breast cancer.[1,2]

Various studies have been conducted on the relationship of the Ki-67 score with clinicopathological parameters in invasive breast carcinomas. [2-7] High Ki-67 index was found significantly related with poor prognostic parameters as high tumor grade and axillary lymph node metastases. [2-5,7]

When the relationship of the Ki-67 index with important prognostic biological markers for breast cancer was investigated, it was found that the high Ki-67 score was associated with hormone receptor (HR) negativity and human epidermal growth factor receptor 2 (HER2) positivity. $[4,5]$ According to their molecular subtypes, the Ki-67 index was found to be higher in Luminal B, HER2 expressing or Triple Negative types compared to Luminal A.[4,7]

In this study, we aimed to evaluate the association of low or high $\mathrm{Ki}-67$ score, using $14 \%$ and $20 \%$ cut-off values, with clinicopathological parameters including age, menopausal status, multifocality-multicentricity (MF/MC), tumor size, histological type, grade, lymphovascular invasion (LVI), perineural invasion, axillary lymph node involvement, estrogen receptor (ER), progesterone receptor (PR), HER2 expression, and molecular subtypes in invasive breast carcinomas.

\section{Materials and Methods}

\section{Patients}

This study included 162 female patients with invasive breast cancer, diagnosed in the pathology department of an university hospital, between April 2011 and May 2012. The patients who were received neoadjuvant therapy were excluded from the study.

Pathology reports were retrospectively reviewed and clinicopathological parameters including age, menopausal status, MF/MC, tumor size, histological type, grade, LVI, perineural invasion, axillary lymph node status, ER, PR, HER2 status, and Ki-67 proliferative index were recorded for each cases.

Cases with multiple tumors were interpreted based on the largest tumor size. Tumor size and axillary lymph node status were classified based on the TNM, AJCC 8 classification.[8] The histological types were evaluated according to the World Health Organization (WHO) Classification of Breast Tumors, $4^{\text {th }}$ Edition.[9] The modified Bloom-Richardson grading system was used for histological grading.[10]

\section{Interpretation of ER, PR, HER2, and Ki-67}

All immunohistochemical studies had been performed in the pathology laboratory of the same university hospital. Cases were considered positive for ER and PR when nuclear staining was observed in at least $1 \%$ of tumor cells regardless of the intensity of staining. ER positivity was also evaluated as two groups: $1-10 \%$ low positive, $>10 \%$ positive (11). HER 2 immunostaining was considered positive when complete intense membrane staining (score $3+$ ) was observed in at least $>10 \%$ of tumor cells.[11] Forty-eight cases which were uncertain for HER2 (score 2) had been evaluated by in situ hybridization and positive 4 cases were also interpreted as HER2 positive.

For Ki-67, CONFIRM anti-Ki67 (30-9) Rabbit Monoclonal Primary Antibody was used. At least 3 fields were selected to represent the spectrum of staining seen on initial overview of the whole section. Scoring involved the counting of at least 500 invasive tumor cells. Nuclear staining was considered positive and staining intensity was not relevant.[1] Then, all cases were divided into groups using $14 \%$ and $20 \%$ cut-off values for Ki-67 score (high: $\geq 14$ and $\geq 20$, low $<14$ and $<20$ ) (Fig. 1). $[12,13]$

\section{Molecular Classification}

All cases were classified into the four molecular subtypes by using HR (ER and/or PR), HER2 and Ki-67 status: Luminal A (HR+ HER2- and low Ki-67 score), Luminal B (HR+ HER2+ or HR+ HER2- and high Ki67 score), HER2 expressing type (HR- HER2+), Triple Negative (HR- HER2-).[12]

\section{Statistical Analysis}

Descriptive statistics were used to describe the data. Normal distribution was tested by Shapiro-Wilk tests.
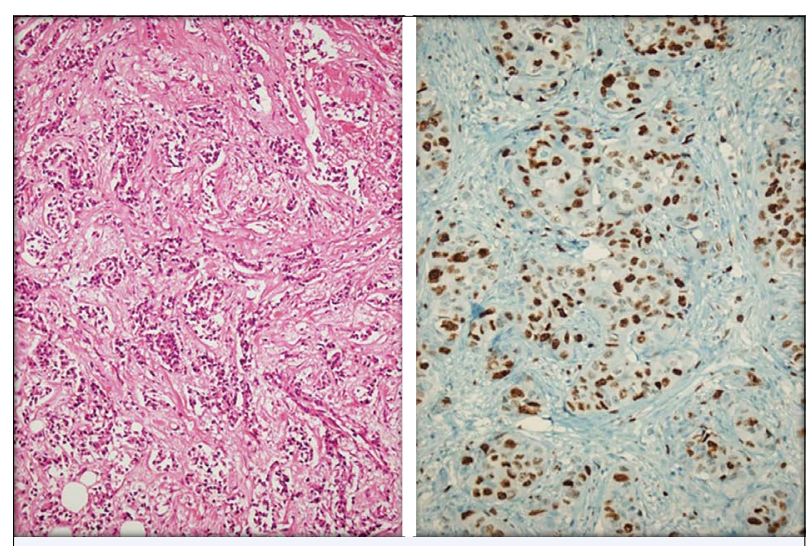

Fig. 1. High Ki-67 score in invasive carcinoma of no special type (Left: H\&E×100, Right: Ki-67×200). 
Non-parametric data were compared using Chi-square test. $\mathrm{P}<0.05$ were considered statistically significant. Statistical analyses were performed using IBM SPSS Statistics for Windows, Version 21.0.

\section{Ethic}

This study is in accordance with the Helsinki Declaration. The study protocol was accepted by Amasya University Clinical Research Ethics Committee (No: 14, Date: 07/01/2021).

\section{Results}

\section{Clinicopathological Characteristics}

The mean age of 162 invasive breast carcinoma cases participating in the study was 55 (24-85) years.

The surgeries performed were breast-conserving surgery in $79(48.8 \%)$ cases, modified radical mastectomy in $65(40.1 \%)$ cases, and simple mastectomy in 18 (11.1\%) cases. Axillary dissections were performed with all breast-conserving surgeries except four cases whose age was over 70 years. In addition, we could not access to follow-up and information of the axillary status of a 48-year-old patient with simple mastectomy.

The tumor sizes varied from 0.4 to 10 (mean 2.6) $\mathrm{cm}$. Thirtyseven $(22.8 \%)$ cases showed MF/MC and 2 (1.2\%) cases had bilateral tumors.

Axillary lymph node status was known for 157 and unknown for 5 (3.1\%) cases. Axillary lymph nodes were negative for $67(41.3 \%)$ cases. $12(7.4 \%)$ cases had micrometastasis (MM) and/or isolated tumor cells (ITC) (MM:7, MM+ITC:2, ITC:3). The remaining 78 (48.2\%) cases contained varying numbers of positive lymph nodes.

According to cut-off value of 14\%: 72 (44.4\%) cases had low and 90 (55.6\%) cases had high Ki-67 score. Molecular subtypes using this value were as follows: 63 (38.9\%) Luminal A, 65 (40.1\%) Luminal B, 13 (8\%) HER2 expressing type, and 21 (13\%) Triple Negative.

According to cut-off value of 20\%: $86(53.1 \%)$ cases had low and 76 (46.9\%) cases had high Ki-67 score. Molecular subtypes using this value were as follows: 74 (45.7\%) Luminal A, 54 (33.3\%) Luminal B, 13 (8\%) HER2 expressing type, and 21 (13\%) Triple Negative. The clinicopathological characteristics of the patients and tumors were summarized in Table 1.

\section{Relationship between Ki-67 Expression and Clini- copathological Parameters}

According to our analysis, the correlations of the Ki-67 score with clinicopathological parameters were simi-
Table 1 Clinicopathological characteristics of invasive breast cancer patients

$\begin{array}{ll}\text { Parameter } & \text { n } \%\end{array}$

Age (years)

$\leq 35$

$36-50$

$14 \quad 8.6$

$51-65$

$43 \quad 26.6$

$>65$

$\begin{array}{ll}70 & 43.2\end{array}$

Menopausal status

$\begin{array}{lll}\text { Premenopausal } & 58 & 35.8\end{array}$

$\begin{array}{lll}\text { Postmenopausal } & 104 & 64.2\end{array}$

Type of surgery

$\begin{array}{lll}\text { Breast-conserving surgery } & 79 & 48.8\end{array}$

$\begin{array}{lll}\text { Modified radical mastectomy } & 65 & 40.1\end{array}$

Simple mastectomy $\quad 18 \quad 11.1$

Laterality

$\begin{array}{lll}\text { Right } & 82 & 50.6\end{array}$

$\begin{array}{lll}\text { Left } & 78 & 48.2\end{array}$

$\begin{array}{lll}\text { Bilateral } & 2 & 1.2\end{array}$

Multifocality/Multicentricity

Present

$37 \quad 22.8$

Absent

$125 \quad 77.2$

Tumor size $(\mathrm{cm})$

$\begin{array}{lll}\leq 2 & 72 & 44.5\end{array}$

$>2$ and $\leq 5 \quad 83 \quad 51.2$

$\begin{array}{lll}>5 & 7 & 4.3\end{array}$

Histological subtype

Invasive carcinoma of no special type (NST) $\quad 114 \quad 70.4$

Mixed invasive NST and lobular carcinoma $\quad \begin{array}{lll}15 & 9.3\end{array}$

Other carcinomas of mixed type $\quad 13 \quad 8.0$

$\begin{array}{lll}\text { Invasive lobular carcinoma } & 8 & 4.9\end{array}$

Metaplastic carcinoma with squamous $\quad 4 \quad 2.5$

differentiation

$\begin{array}{lll}\text { Mucinous carcinoma } & 3 & 1.9\end{array}$

$\begin{array}{lll}\text { Tubular carcinoma } & 2 & 1.2\end{array}$

$\begin{array}{lll}\text { Invasive papillary carcinoma } & 1 & 0.6\end{array}$

Carcinoma with apocrine differentiation $\quad 1 \quad 0.6$

Carcinoma with signet-ring-cell differentiation 10.6

Histological grade

$\begin{array}{lll}\text { I } & 8 & 4.9\end{array}$

II $\quad 82 \quad 50.6$

$\begin{array}{lll}\text { III } & 72 \quad 44.5\end{array}$

Lymphovascular invasion
Present

$\begin{array}{lll}\text { Absent } & 62 & 38.3\end{array}$

Perineural invasion

$\begin{array}{lll}\text { Present } & 70 & 43.2\end{array}$

$\begin{array}{lll}\text { Absent } & 92 & 56.8\end{array}$

Axillary lymph node status
Negative

Micrometastasis and/or ITC $\quad 12 \quad 7.4$

1-3 positive $\quad 46 \quad 28.4$

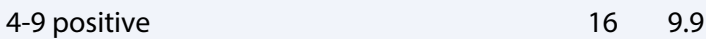




\begin{tabular}{|c|c|c|}
\hline \multicolumn{3}{|l|}{ Table 1 Cont. } \\
\hline Parameter & $\mathbf{n}$ & $\%$ \\
\hline$\geq 10$ positive & 16 & 9.9 \\
\hline Unknown & 5 & 3.1 \\
\hline \multicolumn{3}{|l|}{ Estrogen receptor } \\
\hline Positive & 127 & 78.4 \\
\hline Positive (>10\%) & 121 & 74.7 \\
\hline Low (1-10\%) & 6 & 3.7 \\
\hline Negative & 35 & 21.6 \\
\hline \multicolumn{3}{|l|}{ Progesterone receptor } \\
\hline Positive & 101 & 62.3 \\
\hline Negative & 61 & 37.7 \\
\hline \multicolumn{3}{|l|}{ HER2 } \\
\hline Positive & 24 & 14.8 \\
\hline Negative & 138 & 85.2 \\
\hline \multicolumn{3}{|l|}{ Ki-67 score (14\% cut-off) } \\
\hline Low $(<14 \%)$ & 72 & 44.4 \\
\hline High $(\geq 14 \%)$ & 90 & 55.6 \\
\hline \multicolumn{3}{|l|}{ Ki-67 score ( $20 \%$ cut-off) } \\
\hline Low $(<20 \%)$ & 86 & 53.1 \\
\hline High ( $\geq 20 \%)$ & 76 & 46.9 \\
\hline \multicolumn{3}{|c|}{ Molecular subtypes ( $14 \%$ cut-off) } \\
\hline Luminal $A$ & 63 & 38.9 \\
\hline Luminal B & 65 & 40.1 \\
\hline HER2 expressing type & 13 & 8.0 \\
\hline Triple negative & 21 & 13.0 \\
\hline \multicolumn{3}{|c|}{ Molecular subtypes ( $20 \%$ cut-off) } \\
\hline Luminal $\mathrm{A}$ & 74 & 45.7 \\
\hline Luminal B & 54 & 33.3 \\
\hline HER2 expressing type & 13 & 8.0 \\
\hline Triple negative & 21 & 13.0 \\
\hline Total & 162 & 100.0 \\
\hline
\end{tabular}

NST: No special type; ITC: Isolated tumor cells; HER2: Human epidermal growth factor receptor 2

lar when the cut-off value was $14 \%$ or $20 \%$ (Table 2 ). The Ki-67 score had no relationship with patient age, menopausal status, $\mathrm{MF} / \mathrm{MC}$, and tumor size.

We compared the Ki-67 score with histological types but our case number was not sufficient for statistical analysis. In this study, the most common histological type was invasive carcinoma of no special type (ICNST). They were mostly (56.1\%) had high Ki-67 score based on $14 \%$ cut-off value, but mostly (54.4\%) had low Ki-67 score according to $20 \%$. According to both cut-off values, all (4/4) of the metaplastic carcinomas and carcinoma with signet-ring-cell differentiation (1/1) case had high Ki-67 score, while 7/8 cases of lobular carcinoma and all tubular carcinoma (2/2) cases had low score.

There was a statistically significant association between Ki-67 score and tumor grade $(\mathrm{p}<0.001, \mathrm{p}<0.001)$.
It was observed that grade III cases were more common in the group with high Ki-67.

Ki-67 score was also statistically associated with LVI ( $p=0.002,0.022)$. It was higher in cases with LVI compared to cases with no LVI. There was no association between $\mathrm{Ki}-67$ score and perineural invasion.

For the statistical analysis, five cases of unknown axillary lymph node status were ignored. 157 cases were divided into two groups with negative and positive lymph nodes. Cases with only ITC were added to lymph node-negative group, while the cases with MM were grouped with other lymph node-positive cases. There was no statistically significant relationship between Ki-67 score and axillary lymph node status.

We found a statistically significant association between Ki-67 score and ER status ( $\mathrm{p}=0.001,<0.001)$. $\mathrm{Ki}-67$ was higher in cases that are negative for ER compared to cases which express ER. When we classified the cases as negative, low positive and positive, we found similar results $(\mathrm{p}=0.003, \mathrm{p}=0.001)$. However, since the number of cases in the low positive group was not sufficient, statistical interpretation could not be made regarding the difference between the positive and low positive groups. We could not find any significant correlation of the Ki-67 score with PR and HER2.

When we compared the molecular subtypes, there was a statistically significant association between the Ki-67 score and molecular subtypes $(\mathrm{p}<0.001$, $\mathrm{p}<0.001)$. The Ki-67 score was significantly higher in cases that belong to the Luminal B subtype and lower in cases that belong to Luminal A in comparison to others. Luminal A and B ratios varied according to the cut-off value: All Luminal A cases (63 and 74 cases according to $14 \%$ and $20 \%$ value, respectively) had low $\mathrm{Ki}-67$ score. According to $14 \%$ and $20 \%$ values, respectively, 62 of 65 (95.4\%) and 50 of 54 (92.6\%) Luminal $\mathrm{B}$ cases had high Ki-67 score. Although most cases had high Ki-67 score in Triple-Negative and HER2 expressing subtypes, the rates in these groups were not high enough to make a statistically significant difference in comparison to other molecular subtypes.

\section{Discussion}

In breast cancer, the prognostic significance of the Ki-67 proliferative index has been demonstrated in the majority of the studies.[1,14] Besides, nowadays Ki-67 index is used to predict the response to neoadjuvant chemotherapy in cases with breast cancer.[1,15] It is also used in the differentiation of Luminal A and Luminal B molecular subtypes in invasive breast carcinomas. $[12,16]$ 
Table 2 Analysis of Ki-67 score in relation to clinicopathological parameters of invasive breast cancer patients

\section{Parameter}

\section{Ki-67 score}

\begin{tabular}{|c|c|c|c|c|c|c|c|c|c|}
\hline \multicolumn{5}{|c|}{$14 \%$ cut-off } & \multicolumn{5}{|c|}{$20 \%$ cut-off } \\
\hline \multicolumn{2}{|c|}{ Low } & \multicolumn{2}{|c|}{ High } & \multirow[t]{2}{*}{$\mathbf{p}^{*}$} & \multicolumn{2}{|c|}{ Low } & \multicolumn{2}{|c|}{ High } & \multirow[t]{2}{*}{$\mathbf{p}^{*}$} \\
\hline $\mathbf{n}$ & $\%$ & $\mathbf{n}$ & $\%$ & & $\mathbf{n}$ & $\%$ & $\mathbf{n}$ & $\%$ & \\
\hline
\end{tabular}

\section{Age (years)$$
\leq 35
$$$$
\text { 36-50 }
$$$$
51-65
$$$$
>65
$$

Menopausal status

\section{Premenopausal}

Postmenopausal

Multifocality-multicentricity

$$
\text { Present }
$$

Absent

Grade

I

II

III

Tumor size $(\mathrm{cm})$

$$
\begin{aligned}
& \leq 2 \\
& 2-5 \\
& >5
\end{aligned}
$$

Lymphovascular invasion

Present

Absent

Perineural invasion

$$
\text { Present }
$$

Absent

Lymph node involvement**

Negative

Positive

Estrogen receptor

Positive

Negative

Estrogen receptor

Positive (>10\%)

Low positive (1-10\%)

Negative $(<1 \%)$

Progesterone receptor

$$
\text { Positive }
$$

Negative

HER2 expression

Positive

Negative

Molecular subtype

Luminal A
HER2 expressing
Triple negative

20

$5.6 \quad 10$

11.1

0.116

6

$27.8 \quad 23$

25.6

$27 \quad 37.5$

43

47.8

21

29.2

24
48

48

18

54

47
18$$
36
$$$$
33
$$$$
\begin{gathered}
33 \\
3
\end{gathered}
$$$$
3
$$$$
35
$$$$
37
$$

$33.3 \quad 34$

$\begin{array}{lll}66.7 & 56 & 62.2\end{array}$

34

$25 \quad 19$

75

19

21.1

78.9

9.7

65.3

25

1
35
54

1.1

38.9

60

$50 \quad 36$

$45.8 \quad 50$

4.2

40

55.6

4.4

0.439

$<0.001$

$\begin{array}{lll}48.6 & 65 & 72.2\end{array}$

$\begin{array}{lll}51.4 & 25 & 27.8\end{array}$

27

$$
45
$$

$45 \quad 62.5$

37.5

62.5

$43 \quad 47.8$

$47 \quad 52.2$

$50.7 \quad 35$

$49.3 \quad 53$

39.8

60.2

$\begin{array}{llll}65 & 90.3 & 62 & 68.9\end{array}$

7

9.7

$$
7
$$

4

\section{9}

$\begin{array}{llll}49 & 68.1 & 52 & 57.8\end{array}$

$\begin{array}{llll}23 & 31.9 & 38 & 42.2\end{array}$

$\begin{array}{llll}7 & 9.7 & 17 & 18.9\end{array}$

$\begin{array}{llll}65 & 90.3 & 73 & 81.1\end{array}$

$\begin{array}{llll}63 & 87.5 & 0 & 0\end{array}$

$\begin{array}{llll}3 & 4.2 & 62 & 68.9\end{array}$

$\begin{array}{llll}4 & 5.6 & 9 & 10\end{array}$

$\begin{array}{llll}2 & 2.8 & 19 & 21.1\end{array}$

0.116

24

24
33

7

8

10.5

0.267

$\begin{array}{llll}33 & 38.4 & 37 & 48.7\end{array}$

$\begin{array}{llll}23 & 26.7 & 12 & 15.8\end{array}$

$\begin{array}{lllll}0.558 & 30 & 34.9 & 28 & 36.8\end{array}$

0.795

$\begin{array}{lllll}0.558 & 21 & 24.4 & 16 & 21.1\end{array}$

$\begin{array}{llll}65 & 75.6 & 60 & 78.9\end{array}$

$\begin{array}{cccc}7 & 8.1 & 1 & 1.3 \\ 56 & 65.1 & 26 & 34.2\end{array}$

$\begin{array}{llll}23 & 26.7 & 49 & 64.5\end{array}$

$\begin{array}{llll}4 & 4.7 & 3\end{array}$

$\begin{array}{lllll}0.002 & 46 & 53.5 & 54 & 71.1\end{array}$

0.022

$\begin{array}{lllll}0.189 & 34 & 39.5 & 36 & 47.4\end{array}$

$\begin{array}{llll}52 & 60.5 & 40 & 52.6\end{array}$

$\begin{array}{lllll}0.171 & 39 & 47 & 31 & 41.9\end{array}$

58.1

$\begin{array}{lllll}\mathbf{0 . 0 0 1} & 77 & 89.5 & 50 & 65.8\end{array}$

34.2

$\begin{array}{lllll}\mathbf{0 . 0 0 3} & 73 & 84.9 & 48 & 63.2\end{array}$

0.001

$\begin{array}{llll}9 & 10.5 & 26 & 34.2\end{array}$

$\begin{array}{cccc}9 & 10.5 & 26 & 34.2 \\ 4 & 4.7 & 2 & 2.6\end{array}$

$\begin{array}{lllll}0.180 & 59 & 68.6 & 42 & 55.3\end{array}$

$\begin{array}{llll}27 & 31.4 & 34 & 44.7\end{array}$

$\begin{array}{lllll}0.103 & 10 & 11.6 & 14 & 18.4\end{array}$

0.225

$\begin{array}{llll}76 & 88.4 & 62 & 81.6\end{array}$

$\begin{array}{ccccc}<0.001 & 74 & 86 & 0 & 0 \\ & 4 & 4.7 & 50 & 65.8 \\ & 6 & 7 & 7 & 9.2 \\ & 2 & 2.3 & 19 & 25\end{array}$

${ }^{*} p<0.05$ was considered statistically significant. **Five cases with unknown lymph node status were ignored and 157 cases could be evaluated statistically in terms of lymph node involvement. HER2: Human epidermal growth factor receptor 2 
There is no standardization and a certain cut-point for Ki-67 scoring. The St Gallen consensus 2009 has proposed three scores as low $(\leq 15 \%)$, intermediate (16-30\%), and high (>30\%).[17] According to St Gallen 2011 consensus low Ki-67 score has been defined as $<14 \%$. Based on this cut-off value, HR-positive and HER2 negative breast carcinomas have been classified as Luminal A if they had a low Ki-67 score, and Luminal B if they had a high Ki-67 score.[12] The cut-off value was revised as $20 \%$ at the 2013 St Gallen International Expert Consensus meeting.[13]

In a study, when Ki-67 antibody was applied separately to primary breast carcinoma and synchronous axillary lymph node metastasis, it was shown that there was discordance between Ki-67 expressions. The proportion of Ki-67 labeled cancer cells was significantly higher in axillary metastasis.[18] The method used may also have impact on the significance of Ki-67. In another study, it was found that classical whole section was superior to tissue microarray method in terms of prognosis and clinicopathological correlation.[19] In this study, we only studied on primary breast cancers and excluded the cases with adjuvant therapy. We obtained the Ki-67 proliferative index from archive data and all of them were applied to the whole section during routine work. We used both $14 \%$ and $20 \%$ cut-off values for scoring $\mathrm{Ki}-67 .[12,13]$

The relationship between Ki-67 and clinicopathological parameters in invasive breast carcinomas was investigated in various studies. [2-7] In our study, the correlations of Ki-67 score with clinicopathological parameters were similar when the cut-off value was $14 \%$ or $20 \%$.

Elkablawy et al.[7] reported that high Ki-67 score was statistically associated with older age. When we compared the patient age and menopausal status with the Ki-67 score, we couldn't find a significant relationship.

In this study, the most common histological type was IC-NST and they were mostly had high Ki-67 score based on $14 \%$ cut-off value but mostly had low score according to $20 \%$ cut-off value. We could not perform statistical analysis due to the small number of cases. However, we observed that all cases of the metaplastic carcinoma and carcinoma with signet-ring-cell differentiation cases that known as poor prognostic histological types had high Ki-67 score based on both cut-off values. On the contrary, Ki-67 score was found to be low in most lobular carcinoma and all tubular carcinoma cases, which are known as histological types with a better prognosis than IC-NST.[9]
High Ki-67 score has been reported as associated with poor differentiated tumors.[2] In our study, we found a statistically significant association between $\mathrm{Ki}$ 67 score and tumor grade as in the literature. [2-5,7] We observed that Grade III cases were more common in the group with high Ki-67.

Sun et al.[5] reported that the Ki-67 score increased with increasing tumor size in the early stage breast carcinomas but when the tumor progressed to a certain stage, the Ki-67 score did not increase accordingly. In our study, there was no association between the Ki-67 score and the tumor size.

In some studies, it was reported that there was a significant correlation between Ki-67 score and axillary lymph node involvement.[2,3,7] High Ki-67 score was also reported as associated with distant metastasis.[2] In our series, we could not find a significant association between Ki-67 score and lymph node status. There was also no correlation between the Ki67 score and perineural invasion. Since the data used in the present study were limited to pathology archive reports, distant metastasis data could not be obtained. However, we found that a high Ki-67 score was statistically associated with the presence of LVI. Even if our other results are not enough, we may consider that more invasive tumors had higher Ki-67 score based on this last result.

It has been reported that mean Ki-67 levels and/or Ki-67 score was significantly associated with $\mathrm{HR}$ and HER2 expression. High Ki-67 values had been reported to be correlated with ER negativity, PR negativity, and HER2 positivity.[2,4,5] The present study also exhibited a statistically significant association between Ki-67 score and ER status. Ki-67 was higher in cases which are negative for ER compared to cases which express ER. However, we couldn't find a correlation of Ki-67 score with PR and HER2.

In the literature, it has been reported that the Ki-67 score has a significant relationship with molecular subtypes.[4,7] Aman et al.[4] found that Triple Negative and HER2 expressing subtypes were associated high $\mathrm{Ki}-67$ score. In another study, Ki-67 proliferative index was associated with Luminal B and HER2 expressing type.[7] In our study, we also found a statistically significant association between Ki-67 score and molecular subtypes. Ki-67 score was higher in cases that belong to the Luminal B subtype and lower in cases that belong to Luminal A in comparison to others. The number of cases in Triple-Negative and HER2 expressing types was low. Probably for this reason, although most of the cases had high Ki-67 score in these subtypes, the rates 
were not high enough to make significant difference in comparison to other molecular subgroups.

\section{Conclusion}

In the present study, we obtained results supporting that the Ki-67 score is an important biomarker in breast carcinomas and observed that the correlations of the Ki-67 score with clinicopathological parameters were similar when the cut-off value was $14 \%$ or $20 \%$. We found a significant correlation between high Ki-67 score and high tumor grade, LVI, ER negativity. In addition, the Ki-67 score was higher in cases that belong to the Luminal B subtype and lower in cases that belong to Luminal A in comparison to others. Standardization of interpretation of Ki-67 proliferative index and cut-off value for scoring will improve the demonstration of the prognostic signification of Ki-67 in invasive breast carcinomas.

Peer-review: Externally peer-reviewed.

Conflict of Interest: All authors declared no conflict of interest.

Ethics Committee Approval: This study is in accordance with the Helsinki Declaration. The study was approved by the Amasya University Non-interventional Clinical Research Ethics Committee (No: 14, Date: 07/01/2021).

Financial Support: None declared.

Authorship contributions: Concept - T.Ö.; Design - H.D., T.Ö.; Supervision - H.D., T.Ö.; Funding - None; Materials T.Ö., Ş.İ.; Data collection and/or processing - H.D., T.Ö., Ş.İ;; Data analysis and/or interpretation - H.D., B.T.G.; Literature search - H.D.; Writing - H.D.; Critical review - T.Ö., Ş.İ.

\section{References}

1. Penault-Llorca F, Radosevic-Robin N. Ki-67 assessment in breast cancer: An update. Pathology 2017;49(2):166-71.

2. Ermiah E, Buhmeida A, Abdalla F, Khaled BR, Salem N, Pyrhönen S, et al. Prognostic value of proliferation markers: Immunohistochemical Ki-67 expression and cytometric s-phase fraction of women with breast cancer in Libya. J Cancer 2012;3:421-31.

3. Haroon S, Hashmi AA, Khurshid A, Kanpurwala MA, Mujtuba S, Malik B, et al. Ki-67 index in breast cancer: Correlation with other prognostic markers and potential in Pakistani patients. Asian Pac J Cancer Prev 2013;14(7):4353-8.

4. Aman NA, Doukoure B, Koffi KD, Koui BS, Traore $\mathrm{ZC}$, Kouyate $\mathrm{M}$, et al. Immunohistochemical evaluation of Ki-67 and comparison with clinicopathologic factors in breast carcinomas. Asian Pac J Cancer Prev 2019;20(1):73-9.

5. Sun J, Chen C, Wei W, Zheng H, Yuan J, Tu YI, et al. Associations and indications of Ki-67 expression with clinicopathological parameters and molecular subtypes in invasive breast cancer: A population-based study. Oncol Lett. 2015;10(3):1741-8.

6. Kamranzadeh H, Ardekani RM, Kasaeian A, Sadighi S, Maghsudi S, Jahanzad I, et al. Association between Ki-67 expression and clinicopathological features in prognosis of breast cancer: A retrospective cohort study. J Res Med Sci 2019;24:30.

7. Elkablawy MA, Albasri AM, Mohammed RA, Hussainy AS, Nouh MM, Alhujaily AS. Ki-67 expression in breast cancer. Correlation with prognostic markers and clinicopathological parameters in Saudi patients. Saudi Med J 2016;37(2):137-41.

8. Hortobagyi GN, Connolly JL, D’Orsi CJ, Edge SB, Mittendorf EA, Rugo HS, et al. Breast. In: Amin MB, Edge SB, Greene FL, Byrd DR, Brookland RK, Washington MK, et al, editors. AJCC Cancer Staging Manual. $8^{\text {th }}$ ed. Switzerland: Springer International Publishing AG; 2017. p. 589-628.

9. Lakhani SR, Ellis IO, Schnitt SJ, Tan PH, van de Vijver MJ. WHO Classification of Tumours of the Breast. $4^{\text {th }}$ ed. Lyon, France: IARC Press; 2012.

10. Elston CW, Ellis IO. Pathological prognostic factors in breast cancer. I. The value of histological grade in breast cancer: Experience from a large study with long-term follow-up. Histopathology 1991;19(5):40310.

11. Fitzgibbons PL, Connolly JL. Template for Reporting Results of Biomarker Testing of Specimens from $\mathrm{Pa}-$ tients with Carcinoma of the Breast. CAP Guideline; 2021. Available at: https://www.documents.cap.org/ protocols/Breast.Bmk_1.4.1.0.REL_CAPCP.pdf. Accessed Aug 12, 2021.

12. Goldhirsch A, Wood WC, Coates AS, Gelber RD, Thürlimann B, Senn HJ, Panel Members. Strategies for subtypes--dealing with the diversity of breast cancer: Highlights of the St. Gallen international expert consensus on the primary therapy of early breast cancer 2011. Ann Oncol 2011;22(8):1736-47.

13. Goldhirsch A, Winer EP, Coates AS, Gelber RD, Piccart-Gebhart $M$, Thürlimann $B$, et al. Personalizing the treatment of women with early breast cancer: Highlights of the St Gallen international expert consensus on the primary therapy of early breast cancer 2013 . Ann Oncol 2013;24(9):2206-23.

14. Pérez-López ME, García-Gómez J, Alves MT, Paradela A, García-Mata J, García-Caballero T. Ki-67 is a prognostic marker for hormone receptor positive tumors. Clin Transl Oncol 2016;18(10):996-1002.

15. Jain P, Doval DC, Batra U, Goyal P, Bothra SJ, Agarwal 
C, et al. Ki-67 labeling index as a predictor of response to neoadjuvant chemotherapy in breast cancer. Jpn J Clin Oncol 2019;49(4):329-38.

16. Viale G, Hanlon Newell AE, Walker E, Harlow G, Bai I, Russo L, et al. Ki-67 (30-9) scoring and differentiation of Luminal A- and Luminal B-like breast cancer subtypes. Breast Cancer Res Treat 2019;178(2):451-8.

17. Goldhirsch A, Ingle JN, Gelber RD, Coates AS, Thürlimann B, Senn HJ, Panel Members. Thresholds for therapies: Highlights of the St Gallen international expert consensus on the primary therapy of early breast cancer 2009. Ann Oncol 2009;20(8):1319-29.

18. Kinoe H, Yamanouchi K, Kuba S, Morita M, Sakimura C, Kanetaka K, et al. Discordance of hormone receptor, human epidermal growth factor receptor-2, and Ki-67 between primary breast cancer and synchronous axillary lymph node metastasis. J BUON 2018;23(7):60-6.

19. Dedić Plavetić N, Jakić-Razumović J, Kulić A, Sirotković-Skerlev M, Barić M, Vrbanec D. Prognostic value of ki-67 in breast carcinoma: Tissue microarray method versus whole section analysis-potentials and pitfalls. Pathol Oncol Res 2015;21(2):315-24. 